\title{
Recurrence of Dupuytren's disease (DD) after treatment
}

Hester J. Kan, Frank W. Verrijp, Steven E. R. Hovius, Christianne A. van Nieuwenhoven, Dupuytren Delphi Group, Ruud W. Selles

\section{Source}

Hester J. Kan, Frank W. Verrijp, Steven E. R. Hovius, Christianne A. van Nieuwenhoven, Ruud W. Selles, Dupuytren Delphi Group. (2017). Recurrence of Dupuytren's contracture:

A consensus-based definition. PLOS ONE, vol. 12 (5), e0164849.

An increase in joint contracture in any treated joint of at least 20 degrees at one year post-treatment compared to six weeks post-treatment. 